\title{
Accuracy of railway track conductance and joint efficiency measurement methods
}

\author{
Jacopo Bongiorno ${ }^{1}$, Andrea Mariscotti ${ }^{2}$ \\ ${ }^{1}$ Università di Genova, Via Opera Pia 11A, 16145 Genova, Italy \\ ${ }^{2}$ ASTM Sagl, Via Comacini 7, 6830 Chiasso, Switzerland
}

\begin{abstract}
The IEC 62128-2 (EN 50122-2) indicates methods for the measurement of rail-to-earth conductance and insulating rail joint efficiency in dc electrified railways. These methods are reviewed, considering the influence of system parameters on the accuracy and variability of results. This work reports characterization and quantification of uncertainty and limits of validity, as well as some practical considerations on the execution of measurements. Whereas track conductance is characterized by acceptable uncertainty in the range of one to few \%, insulating joint efficiency test is deemed by a larger and variable uncertainty, so that countermeasures and provisions are necessary.
\end{abstract}

\section{Section: RESEARCH PAPER \\ Keywords: conductivity measurement; DC power systems; electric variables measurement; grounding; guideway transportation power systems; guideway transportation testing; stray current; uncertainty}

Citation: Jacopo Bongiorno and Andrea Mariscotti, Accuracy of railway track conductance and joint efficiency measurement methods, Acta IMEKO, vol. 4, no. 4, article 15, December 2015, identifier: IMEKO-ACTA-04 (2015)-04-15

Editor: Paolo Carbone, University of Perugia, Italy

Received April 12, 2015; In final form October 29, 2015; Published December 2015

Copyright: ㄷ 2015 IMEKO. This is an open-access article distributed under the terms of the Creative Commons Attribution 3.0 License, which permits unrestricted use, distribution, and reproduction in any medium, provided the original author and source are credited

Corresponding author: Andrea Mariscotti, e-mail: andrea.mariscotti@astm-e.ch

\section{INTRODUCTION}

The impact of stray current is mitigated first of all by limiting the rail-to-earth conductance (in the following indicated by $G_{r e}$ when expressed per unit length, so as conductivity) and this clearly stated in the applicable standard for protection against stray current effects in dc systems IEC 62128-2 [1]. The rail-to-earth conductance of a track section depends on several factors, characteristic of the track (type of fasteners and sleepers, use of isolating materials and their performance, type of ballast and sub-ballast, etc.) or external to the system (e.g. type of soil) and also depending on environmental conditions (wet or dry soil, moisture percentage, pollutants, ageing, temperature). All these factors hardly can be thoroughly modelled and accurately predicted, and the measurement assumes a paramount importance, not only to determine if the system meets the limits (dictated by standards or set in the contract), but also the suitability of different provisions and countermeasures.

The control of track conductance is first implemented by dividing the track into electrically independent sections [2]. The track may feature different constructive characteristics depending on the type of support and infrastructure (tunnel, cut \& cover, viaduct, station, etc.), and the provisions adopted for one may not be optimized for the other [3] to [5]. Moreover, such track portions shall in principle allow separate testing, with distinct values of $G_{r e}$. For these reasons mechanical insulating rail joints (IRJs) are used and their correct and satisfactory operation shall be verified, determining the socalled joint efficiency (JE), that is the complement to $100 \%$ of the percentage of rail current leaking through the IRJ itself.

Track isolation and track grounding are two opposite requisites when designing for compliance to two different, but connected, problems: stray current protection and electrical safety of track and wayside installations. The two standards IEC 62128-2 [1] and IEC 62128-1 [6] address them, the former limited to dc systems (stray current is much less relevant for ac systems). To simplify, we might say that corrosion and stray current are relevant in the long term (thus in terms of expected system life, maintenance and monitoring, damage to external third parties), while electrical safety, grounding and bonding, are technical requirements enforced at any time. 
The assessment of the compliance of the design and installation of a new railway system is particularly important in the preliminary stage of a project, when pilot tracks are laid down and construction techniques, as well as provisions and countermeasures, are verified, checking effectiveness, cost, implementation and harmonization. To this aim the experimental determination of track quantities to compare with constraints and prescriptions assumes a primary role: suitability of the measurement technique, embodiment in a standard, accuracy and sensitivity are all factors to consider when preparing the test procedures and the test track setup on site, and when technical discussions between contractors and customer take place.

This work focuses on the measurement methods for track conductance (intended as per-unit-length conductance, or conductivity, measured in $\Omega^{-1} \mathrm{~m}$ ) and joint efficiency appearing in Appendix A of IEC 62128-2 (equivalent to EN 50122-2), considering the spread of results in real conditions for the expected variability of system parameters; technical issues are also considered for the proposed improvements.

\section{MEASUREMENT METHODS APPEARING IN IEC 62128-2}

In this Section the two methods for the measurement of track conductivity and joint efficiency are reviewed, setting the basis for the following analysis and discussion.

\subsection{Standard method for track conductivity measurement}

The measurement of track conductivity is described in App. A, sec. 3, of IEC 62128-2. The method, whose setup is shown in Figure 1, needs two insulating rail joints (IRJs) that electrically separate the running rail.

The standard requires that a DC voltage source is applied across the first IRJ (IRJ-1) and that a nearly constant current flows. The current is proportional to the conductance to earth of the track section under measurement between the two IRJs (with the term "track section" we will identify either a single running rail or both rail, without explicit distinction if it is clear or not determinant).

The quantities that are measured from the test circuit are: the test current $I$ leaving the source terminal, the voltage to ground $V_{P}$ at the voltage terminal located in $\mathrm{P}$. This voltage is in reality measured against a good enough ground connection (e.g. vertical electrode) or the reference grounding circuit, with respect to which the track conductance is to be determined (e.g. concrete mesh, stray current collector, etc.).

Due to contingencies and practical issues, the measurement execution may be subject to some constraints, related to: availability of a long enough track behind the source IRJ (IRJ-1) or that this track is solidly grounded to close the measurement circuit; the voltage terminal within the section may be moved

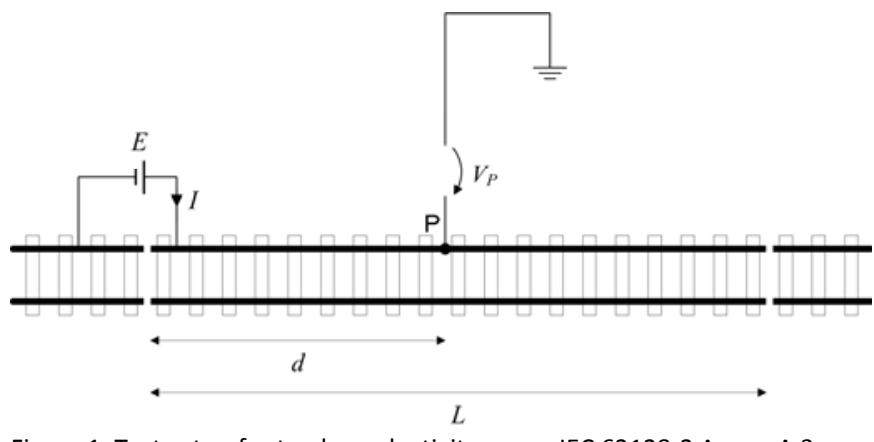

Figure 1. Test setup for track conductivity as per IEC 62128-2 Annex A.3. along it, to ease access to the reference ground potential point, and - it is observed - its position is subject to a minimum distance $d_{\min }=50 \mathrm{~m}$ from the first IRJ, but no maximum length is prescribed. The standard indicates also a maximum length of the section $L_{\max }=2 \mathrm{~km}$. Both constraints are aimed at impeding too a favourable configuration, that would lead to underestimation of track conductivity as we will see in Section 3.1. The injection point distance from IRJ $x$ is not constrained, but it is reasonable to assume that it is quite close to the IRJ under test; by the way, it is possible to show that changing it does not influence appreciably the total amount of current flowing in the left and right rail sections in parallel.

$Z_{x, l} \cong \frac{1}{G_{r e} X} \quad Z_{x, r} \cong \frac{1}{G_{r e}(L-x)} \quad Z_{i n j}=Z_{x, l} / / Z_{x, r}$.

Equations in (1) describe the relationship between equivalent impedances seen by injection point, respect right and left parts of the system, and it is evident that $Z_{i n j}$ keeps almost constant with the left term reducing and the right term increasing, while $x$ moves from left (minimum distance from origin) to right (nearly full length $L$ ).

The conductivity to earth is estimated, following indications in IEC 62128-2 as

$G_{r e}^{\prime}=\frac{I}{V_{P} L}$,

where $I$ is the total current at the injection point, $L$ is the section length, $V_{P}$ is the voltage at the point $\mathrm{P}$.

\subsection{Standard method for joint efficiency measurement}

The method for the measurement of joint efficiency appears in App. A, sec. 5, of IEC 62128-2. The method, whose setup is shown in Figure 2, needs two insulating rail joints (IRJs): one is the joint under test (JUT), the other one at the other end electrically separates the rail section.

A DC voltage source $E$ is applied across the JUT IRJ-1 and the current flowing behaves as described at the beginning of Section 2. The standard specifies that two longitudinal voltage drops across $10 \mathrm{~m}$ of rail are measured at the left and the right of the injection point and the ratio of the voltages gives the joint efficiency (JE) (3). Before using the measured voltages, they are corrected for the off-voltages, i.e. the voltages measured once the source voltage is switched-off.

$$
J E=\frac{U_{2, \text { on }}-U_{2, \text { off }}}{\left(U_{1, \text { on }}-U_{1, \text { off }}\right)+\left(U_{2, \text { on }}-U_{2, \text { off }}\right)} \times 100 \% \text {. }
$$

In reality, because the longitudinal resistance of the rail is a constant, the same equation holds also for the current terms flowing in the two voltmetric sections.

The limit for JE established by the standard is $95 \%$.

The length $L_{V}=10 \mathrm{~m}$ for the measurement of the longitudinal voltage drop is dictated by the standard, as well as

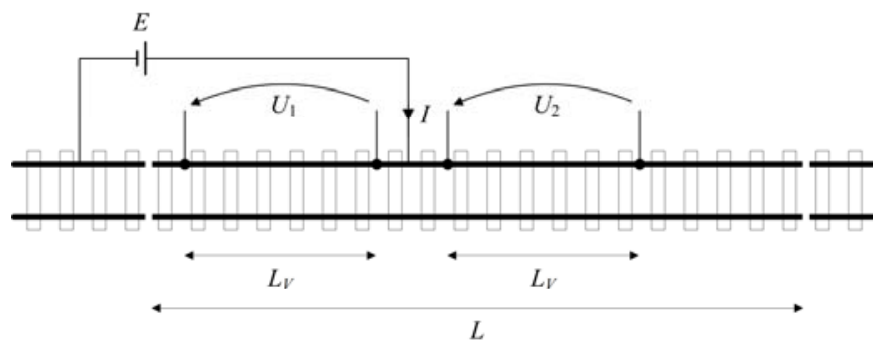

Figure 2. Test setup for joint efficiency as per IEC 62128-2 Annex A.5. 
the position of the current injection point, immediately after the first two voltage terminals, that is at a distance slightly larger than $10 \mathrm{~m}$. While the resulting JE is not influenced by $G_{r e}$, the value of $L_{V}$ is quite relevant.

\section{VARIABILITY OF RESULTS}

The expressions reported in the previous Section are now analyzed with respect to plausible parameter variations, solving an equivalent circuit which represents the considered system, in order to check their validity and the spread of results.

\subsection{Track conductance per-unit-length}

The expression for the calculation of per-unit-length track conductance from raw measurements (2) relies implicitly on the fact that the whole test current $I$ flows past the voltage terminal, so that the only correction to apply suggested in the standard is for the voltage drop in the running rail. The effect of the fraction of the test current leaving the rail through the conductance-to-earth of the left section before the voltage terminal at $\mathrm{P}$ is not taken into account (see Figure 3). If the distance of the voltage terminal $d$ from IRJ-1 were much less than the total track section $L$, then this phenomenon represents a minor source of error.

The rail section is modelled as a ladder network of resistance and conductance elements, $R_{r i}$ and $G_{r i}$, respectively, each determined by the per-unit-length values multiplied by the equivalent length of the circuit cell; $R_{0}$ represents the equivalent resistance of track sections behind IRJ-1. The equivalent circuit may be simplified for any point $\mathrm{P}$ by calculating the Thevenin equivalent $\left(E_{\mathrm{P}}\right.$ in series to $Z_{\mathrm{P}}^{\prime}$ ) for the left part (going iteratively from the source to the point $\mathrm{P}$ ) and the series-parallel equivalent resistance $\left(Z^{\prime \prime}{ }_{\mathrm{p}}\right)$ for the right part (going backwards from the last element $N$ to the point P). The details of calculations are omitted.

The variability of the estimated conductivity with section length $L$ and position $\mathrm{P}$ of the voltmetric measurement point is analyzed in Figure 4.

The attention is focused on five different track-section lengths $L$, spanning from a minimum of $250 \mathrm{~m}$ (typical of depots and some stations with short trains, such as metro and light railways) to the maximum allowed by the standard $L_{\text {max }}=2 \mathrm{~km}$. Rail resistivity, ground conductivity and $R_{0}$, used in the calculation are assumed and kept constant. The equivalent circuit is solved moving the voltmetric measurement position $\mathrm{P}$ and varying the distance from the IRJ from the minimum $d_{\text {min }}=50 \mathrm{~m}$ to a practical maximum distance corresponding to half of the length $L$. The estimated conductivity $G_{r e}^{\prime}$ decreases with $L$ and increases with $d$. Assuming an absolute validity of the estimated $G_{r e}^{\prime}$ independent on $L$, the spread of values at the minimum distance of $50 \mathrm{~m}$ is nearly $1 \%$ and slightly smaller moving away from the injection point, i.e. with the distance of the voltage terminal $d>d_{\text {min }}$. The dependency on position $\mathrm{P}$ is limited to about $0.2 \%$ relative for

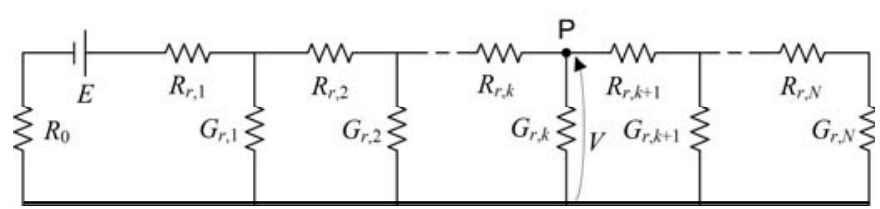

Figure 3. Equivalent circuit of the rail section between IRJs with voltage source $E$ across IRJ-1 and voltmetric terminal at point $P$; the reference potential common to all conductance elements and $R_{0}$ is ground.

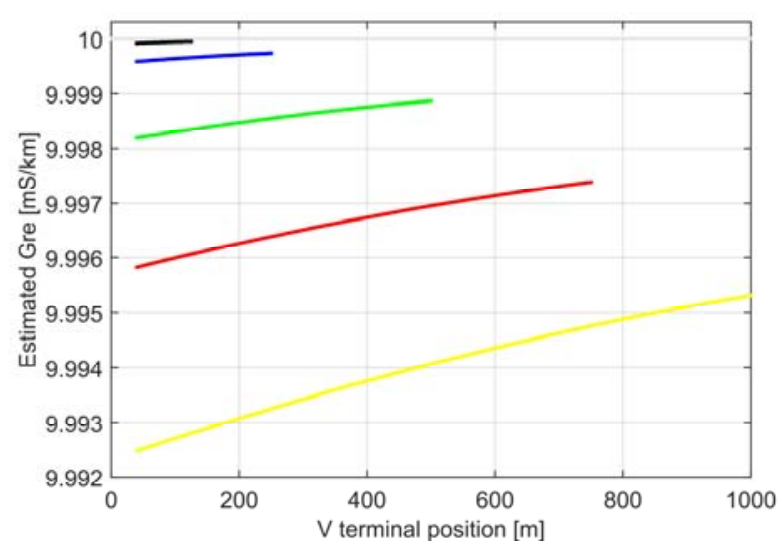

(a)

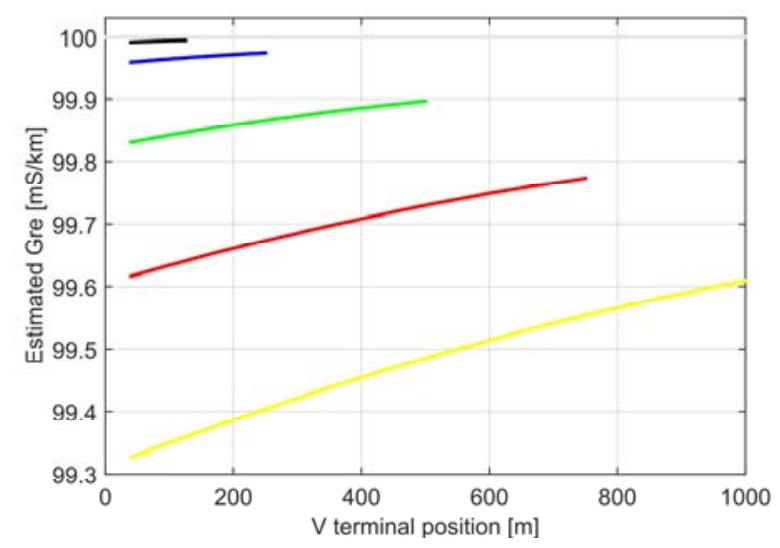

(b)

Figure 4. Estimated conductivity for various test section length values $L$ (from top to bottom: $250 \mathrm{~m}$ black, $500 \mathrm{~m}$ blue, $1000 \mathrm{~m}$ green, $1500 \mathrm{~m}$ red, $2000 \mathrm{~m}$ yellow) vs. the position $\mathrm{P}$ of the voltmetric $V$ terminal (that moves from $50 \mathrm{~m}$ up to $L / 2$ ): rail conductivity $G_{r e}$ is (a) $10 \mathrm{mS} / \mathrm{km}$, (b) $100 \mathrm{mS} / \mathrm{km}$, $R_{0}=1 \Omega, R_{r}=20 \mathrm{~m} \Omega / \mathrm{km}$.

each $L$.

The tracks behind the IRJ under test (IRJ-1) and closing the circuit for the current to flow through the soil are modelled with an equivalent resistance $R_{0}$ that is in series to the track section subject to measurement. The influence is evaluated in Figure 5 , where $R_{0}$ ranges from $0.1 \Omega$ to $20 \Omega$ and the estimated $G_{r e}^{\prime}$ values are plotted for three different track section

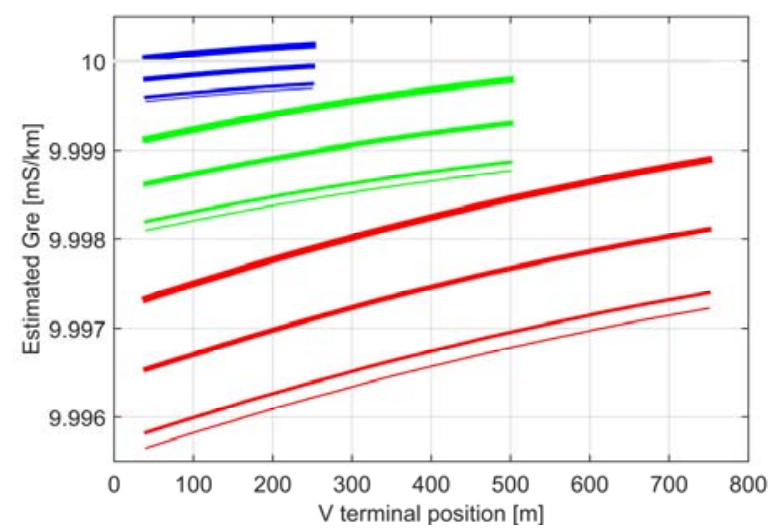

Figure 5. Estimated conductivity as a function of the assumed equivalent resistance $R_{0}$ of tracks behind IRJ-1 vs. varying voltmetric $V$ terminal position $\mathrm{P}$ between $50 \mathrm{~m}$ and $L / 2: R_{0}=0.1 \Omega, 1 \Omega, 10 \Omega$ and $20 \Omega$ (thinner to thicker in each group of four curves); $G_{\mathrm{re}}=10 \mathrm{mS} / \mathrm{km}, R_{\mathrm{r}}=20 \mathrm{~m} \Omega / \mathrm{km}, L=$ $500 \mathrm{~m}$ (blue), $1000 \mathrm{~m}$ (green) and $1500 \mathrm{~m}$ (red). 
lengths $L=500 \mathrm{~m}, 1000 \mathrm{~m}$ and $1500 \mathrm{~m}$. Low values of $R_{0}$ indicate a well earthed long track section behind the first joint; conversely large values are typical of short track sections (maybe accidentally sectioned) and hence a more difficult path for the return current of the source.

In Figure 5 it can be seen that for short test sections and large $R_{0}$ values the estimated $G_{r e}^{\prime}$ may be larger than the real one, but in general the error is always to reduce the estimated $G_{r e}^{\prime}$ that is on the wrong side with respect to limits. However, the error is always well below $1 \%$ and $R_{0}=20 \Omega$ represents already a worst case.

The estimated conductivity depends slightly also on the rail longitudinal resistance $R_{r}$, that causes a longitudinal voltage drop due to the flowing test current: this is investigated in Figure 6 , where three track lengths $L$ are considered fixing $R_{0}=5 \Omega$ and $G_{r e}=10 \mathrm{mS} / \mathrm{km}$. It is observed that the estimated $G_{r e}^{\prime}$ is larger when the rail resistance $\mathrm{R}_{r}$ is smaller; the used $\mathrm{R}_{r}$ values are characteristic of large- and medium-gauge running rails and the resulting spread of values is around $1 \%$.

\subsection{Joint efficiency}

Assuming an ideal IRJ with infinite resistance, the application of (3) for different $L_{V}$ brings to values of JE (in principle $100 \%$ ) that are easily as low as $85 \%$ to $90 \%$, thus leading to a failed test and a rejected IRJ. We call this as "setup limit of JE" and cannot be lower than $95 \%$; moreover, it should be higher than that, in order to accommodate some tolerance for less-than-ideal joints, measurement uncertainty and round-offs.

It is underlined that while the resistance of a joint is a unique value, joint efficiency depends also on track characteristics: a joint isolates a rail section better if the rail-to-earth conductance is large.

A simulation with the simple equivalent circuit shown in Figure 3 was done for $L_{V}=2.5 \mathrm{~m}, 5 \mathrm{~m}, 10 \mathrm{~m}$ and $20 \mathrm{~m}$ and the results are reported in Figure 7.

Having reduced the length of the voltmetric sections to $5 \mathrm{~m}$, first, and then to $2.5 \mathrm{~m}$, the measured efficiency was increased above the limit of $95 \%$. However, reducing $L_{V}$ has the undesired effect of reducing the amplitude of the measured drops $U_{1}$ and $U_{2}$, reducing the signal-to-noise ratio and going quite close to the sensitivity of the used instrumentation. Preexisting voltages and offsets may be easily compensated for.

With $L_{V}=10 \mathrm{~m}$ as prescribed, to bring the setup limit of JE

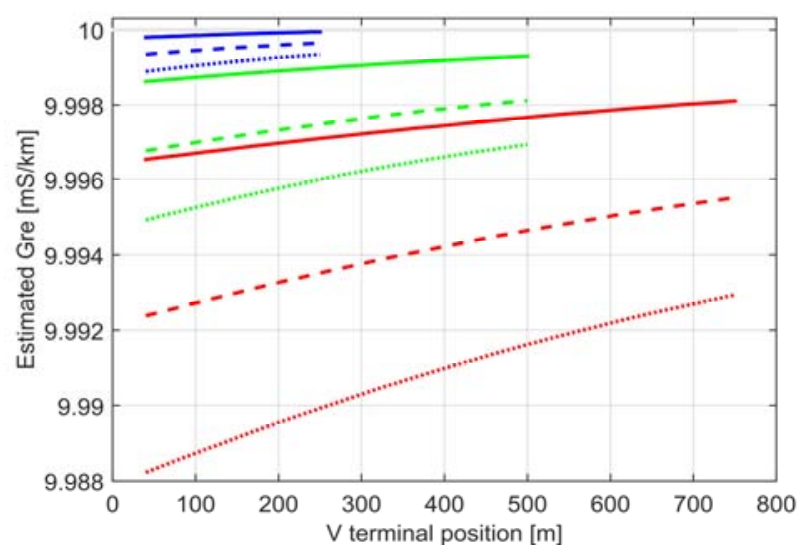

Figure 6. Estimated conductivity for different rail longitudinal resistance values $R_{\mathrm{r}}=20$ (solid), 40 (dashed), 60 (dotted) $\mathrm{m} \Omega / \mathrm{km}$ vs. voltmetric $V$ terminal position $P$ between $50 \mathrm{~m}$ and $L / 2: G_{\mathrm{re}}=10 \mathrm{mS} / \mathrm{km}, L=500 \mathrm{~m}$ (blue), $1000 \mathrm{~m}$ (green) and $1500 \mathrm{~m}$ (red), $R_{0} 5 \Omega$.
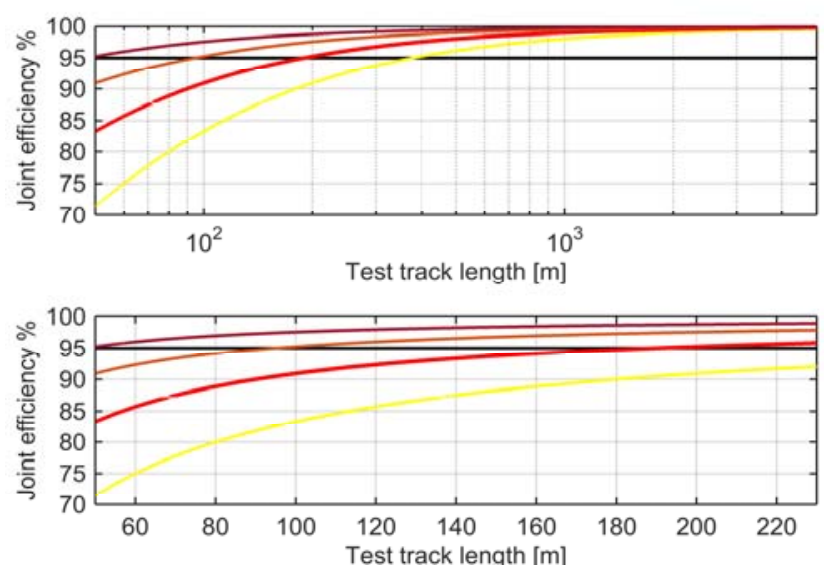

Figure 7. Joint Efficiency as resulting from the application of IEC 62128-2 equation and for three different values of $L_{\mathrm{V}}: 2.5 \mathrm{~m}$ and $5 \mathrm{~m}$ (brown upper curves), $10 \mathrm{~m}$ (thick red curve), $20 \mathrm{~m}$ (yellow lower curve). $R_{0}=10 \Omega, R_{\mathrm{r}}=$ $35 \mathrm{~m} \Omega / \mathrm{km}, G_{\mathrm{re}}=10 \mathrm{mS} / \mathrm{km}$.

above $95 \%$, the required rail section length $L$ shall be at least $200 \mathrm{~m}$. In practical situations this may not be feasible, as for shunting yards and depots of light railways and metros, where trains are never very long and turnouts and shunts are often separated by $150 \mathrm{~m}$ to $200 \mathrm{~m}$ of track.

Another practical factor that may in turn limit the reduction of $L_{V}$ is the safety requirement of using SELV voltage, which for $\mathrm{dc}$ is $60 \mathrm{~V}$. For a good rail with low conductivity to ground (e.g. $10 \mathrm{mS} / \mathrm{km}$ or lower [7], [8]) this would imply $0.48 \mathrm{~A} / \mathrm{km}$, or $48 \mathrm{~mA}$ every $100 \mathrm{~m}$. With a longitudinal rail resistance in the range of $20 \mu \Omega / \mathrm{m}$ to $50 \mu \Omega / \mathrm{m}$, the resulting voltage drop across the voltage terminals separated by $L_{V}$ meters, would be 9.6 $\mu \mathrm{V}$ to $24 \mu \mathrm{V}$ reading for $L_{V}=10 \mathrm{~m}$ and $L=200 \mathrm{~m}$, reducing to $4.8 \mu \mathrm{V}$ to $12 \mu \mathrm{V}$, when $L_{V}$ is reduced to $5 \mathrm{~m}$. A microvoltmeter and repeated readings shall be adopted, in order to reduce unavoidable fluctuations; it is observed that offvoltages are large enough to affect the accuracy of such readings. It is underlined that the intensities of injected current suggested by the standard are not attainable if the SELV voltage limit is enforced; to reach some Ampère of intensity for moderate track-section length, the voltage of the dc source shall be increased to more than a hundred volts.

The problem is now considered the other way round, that is by assigning IRJs a known resistance $(1 \mathrm{k} \Omega, 10 \mathrm{k} \Omega$ and $100 \mathrm{k} \Omega$, simulating an increasing efficiency) and a sensitivity study is made to identify which parameters influence the so-determined JE value, causing the "setup limit of JE" (see Figure 8).

For high-efficiency IRJs (i.e. with equivalent resistance larger than $100 \mathrm{k} \Omega$ ) large track-conductivity values do not interfere with the test and the resulting $J E$ values are sufficiently large, provided that the test track is long enough, i.e. longer than about $200 \mathrm{~m}$ minimum. On the contrary, less efficient IRJs cannot be satisfactorily measured and variability of track conductance has a negative impact on test accuracy.

\section{OTHER PRACTICAL CONSIDERATIONS}

Besides the evaluation of expressions, also the measurement methods are considered, as far as their applicability and possible improvements are concerned.

\subsection{DC source and extraneous voltages}

It is required to use a dc voltage source. This is quite convenient for the availability of dc voltages of $12 \mathrm{~V}, 24 \mathrm{~V}$ and 

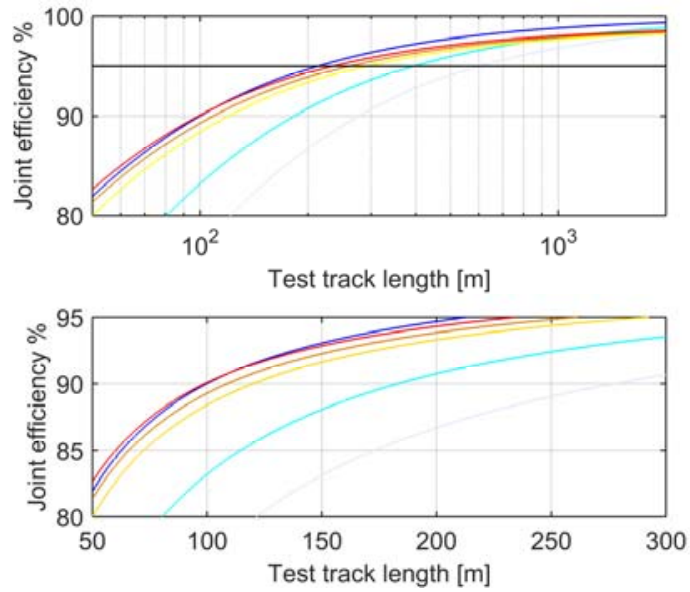

Figure 8. Joint Efficiency with real IRJs of finite resistance: $5 \mathrm{k} \Omega, 10 \mathrm{k} \Omega$, $\geq 100 \mathrm{k} \Omega$ (from lighter to darkest line); hot and cold colours refer to $G_{r e}=10 \mathrm{mS} / \mathrm{km}$ and $100 \mathrm{mS} / \mathrm{km}$, respectively; $R_{0}=10 \Omega, R_{r}=35 \mathrm{~m} \Omega / \mathrm{km}$.

$48 \mathrm{~V}$ from batteries of any size, freeing the operator from mains sockets. DC measurements, however, are normally affected by extraneous voltages developing because of electrochemical reactions in the soil and junctions between different metals. Two consecutive readings with polarity reversal is the usually adopted solution; the standard unfortunately does not include such possibility, in favour of a simpler additional reading in off conditions, aiming to tackle the problem of pre-existing extraneous voltages. This technique, in principle, is not able to reproduce the same conditions that occur during on-time intervals, when the source voltage is applied and current is flowing. However, practically speaking, off-voltage and reversed polarity readings are quite in agreement, based on experimental observations. For example, for a track-conductance measurement the two positive and negative voltages were $+50.4 \mathrm{~V}$ and $-49.9 \mathrm{~V}$, with an offvoltage of $0.44 \mathrm{~V}$, so quite close to their difference.

\subsection{Polarization}

When applying dc voltage in the "on" interval, quite rapidly polarization occurs in the soil and in the other electrolytic substances that may be dissolved in water and moisture trapped beneath the rails and in the fastening system. When the source voltage is disconnected (as rapidly as possible), the voltage readings during the "off" interval are quite critical. The standard does not say anything for the track-conductivity measurement, but for the joint-efficiency measurement it specifies "directly after the switching off'. Practical experience confirms that this might be interpreted by the operator as "a fraction of a second" or "some seconds" after switching off, depending also on his/her speed in storing values, taking notes, etc.. When a data logger is used minimizing delays, it may be observed that the off-voltage due to polarization varies rapidly in the first 3 to 5 seconds, with the exact time instant of the measurement representing a source of uncertainty. When measuring joint efficiency at very low voltage values, the off voltages that are subtracted from the on voltages may become a significant percentage, ranging on average from some $\%$ to up to $30 \%$. Varying of a few seconds the instant of off-voltage reading has an overall impact on the final result of about $1 \%$ to $15 \%$ based on experience. In extreme cases the "off" voltage may be nearly equal to the "on" voltage. In Table 1 there are reported sample readings made at low temperature $\left(3{ }^{\circ} \mathrm{C}\right.$ to $4{ }^{\circ} \mathrm{C}$ ), with wet soil conditions and humidity around $50 \%$ for
Table 1. Polarization for various joint efficiency measurements.

\begin{tabular}{|c|c|c|c|c|c|}
\hline & & \multicolumn{2}{|c|}{$U_{1}[\mathrm{mV}]$} & \multicolumn{2}{|c|}{$U_{2}[\mathrm{mV}]$} \\
\hline & & $2 s$ & $5 s$ & $2 s$ & $5 s$ \\
\hline \multirow{2}{*}{ meas. 1} & on & \multicolumn{2}{|c|}{0.020} & \multicolumn{2}{|c|}{0.290} \\
\hline & off & 0.006 & 0.003 & 0.044 & 0.025 \\
\hline \multirow{2}{*}{ meas. 2} & on & \multicolumn{2}{|c|}{0.021} & \multicolumn{2}{|c|}{0.187} \\
\hline & off & 0.016 & 0.012 & 0.040 & 0.042 \\
\hline \multirow{2}{*}{ meas. 3} & on & \multicolumn{2}{|c|}{0.027} & \multicolumn{2}{|c|}{0.172} \\
\hline & off & 0.011 & 0.08 & 0.030 & 0.022 \\
\hline \multirow{2}{*}{ meas. 4} & on & \multicolumn{2}{|c|}{0.012} & \multicolumn{2}{|c|}{0.095} \\
\hline & off & 0.011 & 0.06 & 0.012 & 0.09 \\
\hline
\end{tabular}

the first three measurements (due to soft rain the day before) and the fourth one with some drier soil (measurement taken the following day).

Observing the values reported for $2 \mathrm{~s}$ and $5 \mathrm{~s}$ interval after switching off, various behaviours may be outlined: voltage drops by $50 \%$ (meas. 1), by only $30 \%$ (meas. 3), in between these values (meas. 4) or does not appreciably change (meas. 2). This means that there is no rule to follow to compensate for off-voltage readings taken accidentally at different instants of time and that the method of measurement should be more detailed.

\section{CONCLUSIONS}

The methods were considered for the experimental determination of per-unit-length track conductance to earth and joint efficiency, as reported in the IEC 62128-2 standard. This standard was reiterated until the most recent version in 2012, so that the methods may be considered mature.

It was shown that the track-conductance method exhibits a variability with respect to the considered parameters (length of test section, rail resistance, rail-to-earth conductivity and resistance-to-earth of the tracks behind the test section) that is limited to $1 \%$, in general causing underestimation of the real conductance value.

For the joint efficiency method the variability is much larger and the method itself in many cases (especially for short test sections of less than $200 \mathrm{~m}$ ) is unable to determine satisfactorily joint efficiency, if the limit of $95 \%$ given in the IEC 62128-2 is considered, thus causing rejection of good rail joints. The analysis shows which parameters of the setup influence the effectiveness of the method. However, the offered solution of reducing the separation of voltmetric terminals on the two sides of the current injection point reduces the voltage drop intensity, and shall be evaluated against the non-negligible intensity of disturbing external voltages and polarization. The off-voltage reading after the "on" phase is subject to rapid variations in the first seconds after switch off: this is a relevant source of uncertainty and the standard does not establish a clear method, nor the exact amount of time to elapse after switch off to take off-voltage readings.

When the length of the test track section is such to cause unavoidable and relevant error, it is proposed to adopt a combination of the following: reduce the voltmetric terminal separation to $5 \mathrm{~m}$ and increase the supply voltage to $100 \mathrm{~V}$.

\section{REFERENCES}

[1] IEC 62128-2, Railway applications - Fixed installations Electrical safety, earthing and the return circuit - Part 2: Provisions against the effects of stray currents caused by d.c. traction systems, 2012. 
[2] K. S. Bahra and R. E. Catlow, "Control of Stray Currents for D.C. Traction Systems," IEE Conference Publication No. 405, Electric Railways in a United Europe, 27-30 March 1995.

[3] D. Paul, "DC Traction Power System Grounding," IEEE Transactions on Industrial Applications, Vol. 38, no. 3, 2002, pp. 818-824.

[4] A. Ogunsola, A. Mariscotti and L. Sandrolini, "Estimation of Stray Current from a DC Electrified Railway and Impressed Potential on a Buried Pipe", IEEE Transactions on Power Delivery, Vol. 27 no. 4, Oct. 2012, pp. 2238-2246.

[5] I. Cotton, C. Charalambous, P. Aylott, and P. Ernst, "Stray Current Control in DC Mass Transit Systems," IEEE
Transactions on Vehicular Technology, Vol. 54, no. 2, March 2005, pp. 722-730.

[6] IEC 62128-1, Railway applications - Fixed installations Electrical safety, earthing and the return circuit - Part 1: Protective provisions against electric shock, 2012.

[7] Shi-Lin Chen, Shih-Che Hsu, Chin-Tien Tseng, Kun-Hong Yan, Huang-Yu Chou, and Tong-Ming Too, "Analysis of Rail Potential and Stray Current for Taipei Metro," IEEE Transactions on Vehicular Technology, Vol. 55, no. 1, Jan. 2006, pp. 67-75.

[8] A. Mariscotti and P. Pozzobon, "Experimental results on low rail-to-rail conductance values", IEEE Transactions on Vehicular Technology, vol. 54, n. 3, May 2005, pp. 1219-1222. 